\title{
Neoadjuvant therapy in locally advanced colon cancer: a meta- analysis and systematic review
}

\author{
Chin Kai Cheong ${ }^{1}$, Kameswara Rishi Yeshayahu Nistala ${ }^{1}$, Cheng Han $\mathbf{N g}^{1}$, Nicholas Syn ${ }^{1}$, \\ Heidi Sian Ying Chang ${ }^{2}$, Raghav Sundar ${ }^{1,3,4}$, Soon Yu Yang ${ }^{5}$, Choon Seng Chong ${ }^{1,2}$ \\ ${ }^{1}$ Yong Loo Lin School of Medicine, National University of Singapore, Singapore, Singapore; ${ }^{2}$ Department of Surgery, University Surgical Cluster, \\ National University Hospital, Singapore, Singapore; ${ }^{3}$ Department of Haematology-Oncology, National University Cancer Institute, Singapore \\ (NCIS), National University Health System, Singapore, Singapore; ${ }^{4}$ The N.1 Institute for Health, National University of Singapore, Singapore, \\ Singapore; ${ }^{5}$ Department of Radiation Oncology, National University Cancer Institute, Singapore (NCIS), National University Health System, \\ Singapore, Singapore \\ Contributions: (I) Conception and design: All authors; (II) Administrative support: CK Cheong, KRY Nistala, CH Ng, N Syn; (III) Provision of study \\ materials or patients: CK Cheong, KRY Nistala, CH Ng, CS Chong; (IV) Collection and assembly of data: CK Cheong, KRY Nistala, CH Ng, N \\ Syn; (V) Data analysis and interpretation: All authors; (VI) Manuscript writing: All authors; (VII) Final approval of manuscript: All authors. \\ Correspondence to: Raghav Sundar; Choon Seng Chong. Yong Loo Lin School of Medicine, National University of Singapore, 5 Lower Kent Ridge \\ Rd., Singapore 119074, Singapore. Email: raghav_sundar@nuhs.edu.sg; choon_seng_chong@nuhs.edu.sg.
}

Background: The role of perioperative or neoadjuvant chemotherapy for locally advanced colon cancer is unclear. Emerging evidence such as the FOXTROT trial is challenging the conventional norm of upfront operation for these patients. However, these trials have yet to reach statistical significance.

Methods: MEDLINE, Embase, Cochrane Library, China Knowledge Resource Integrated Database (CNKI) and ClinicalTrials.gov were searched. Randomized controlled trials (RCTs) and observational studies of patients with locally advanced colon cancer were included. The intervention arm was neoadjuvant chemotherapies while the comparator arm was adjuvant chemotherapies. Studies which reported outcomes of interests included overall survival, disease-free survival, R0 resection rate, perioperative complications and adverse effects of chemotherapy were chosen.

Results: We identified five eligible randomized trials and two observational studies, including 29,504 patients. Neoadjuvant therapies exhibited statistically significant improvement in overall survival [hazard ratio $(\mathrm{HR})=0.76,95 \%$ confidence interval $(\mathrm{CI}): 0.65-0.89, \mathrm{P}=0.0005]$, and disease-free survival $(\mathrm{HR}=0.74$, 95\% CI: $0.58-0.95, \mathrm{P}=0.02)$. R0 resection rate fell slightly short of significance [odds ratio $(\mathrm{OR})=1.86,95 \%$ CI: $0.95-3.62, \mathrm{P}=0.07]$. Risk of peri-operative complications did not differ between groups when examining abdominal infection [risk ratio $(\mathrm{RR})=1.14,95 \% \mathrm{CI}: 0.59-2.18, \mathrm{P}=0.70$ ] and anastomotic leakage $(\mathrm{RR}=0.83$, 95\% CI: 0.53-1.31, P=0.42). No statistical differences in complications from chemotherapy were reported.

Conclusions: This meta-analysis highlights the potential survival benefit of neoadjuvant chemotherapy compared to adjuvant chemotherapy for locally advanced colon cancer, without an increase in surgical morbidity. Neoadjuvant or perioperative approaches may be considered an alternative to upfront surgery followed by chemotherapy for locally advanced colon cancer.

Keywords: Neoadjuvant therapy; colonic neoplasms; meta-analysis

Submitted May 29, 2020. Accepted for publication Aug 17, 2020.

doi: 10.21037/jgo-20-220

View this article at: http://dx.doi.org/10.21037/jgo-20-220 


\section{Introduction}

Colon cancer is a leading cause of morbidity and mortality worldwide (1). The mainstay treatment of non-metastatic colon cancer is surgery with curative intent. For locally advanced disease (high-risk stage II and stage III), treatment with adjuvant chemotherapy after surgery is recommended (2). Neoadjuvant systemic therapy approaches are considered standard-of-care in several other gastro-intestinal tumor types such as gastric, esophageal and rectal cancer (3-5). There are several benefits of neoadjuvant systemic therapy: (I) improvement of surgical outcomes by downstaging of tumor; (II) early control of systemic metastatic spread and test of tumor biology; and (III) in vivo test of chemotherapy sensitivity and potential incorporation of novel agents in clinical trials. Apart from these benefits, trials from other tumor types have demonstrated that there is no decrease in survival by the early introduction of chemotherapy $(5,6)$. However, few perioperative or neoadjuvant chemotherapy trials have been conducted in colon cancer. Smaller phase II studies have demonstrated the safety of neoadjuvant chemotherapy, but few trials have the statistical power to detect a difference in survival (7-9). The recent FOXTROT trial (10) presented at ASCO June 2019, reported better surgical outcomes but did not meet statistical significance for survival benefit at an early followup of 2 years. We evaluated the outcomes of neoadjuvant versus adjuvant chemotherapy in locally advanced, nonmetastatic colon cancer by means of a systematic review and meta-analysis. We present the following article in accordance with the PRISMA reporting checklist (available at http://dx.doi.org/10.21037/jgo-20-220).

\section{Methods}

A systematic review was performed in accordance with the Preferred Reporting Items for Systematic Reviews and Meta-Analyses (PRISMA) guidelines (11).

\section{Search strategy}

MEDLINE, Embase, Cochrane Library and China Knowledge Resource Integrated Database (CNKI) (12) electronic databases were searched from their inception to 4 May 2020 with assistance from a medical librarian. In addition, searches were conducted on ClinicalTrials.gov for ongoing studies and hand searching of references in included studies. The search strategy is documented in the Supplementary. All potential studies were imported into
EndNote $\mathrm{X} 9$ and duplicates removed.

\section{Inclusion criteria and exclusion criteria}

Inclusion criteria were: (I) studies that involved patients who had locally advanced colon cancer; (II) studies comparing the effects of neoadjuvant or perioperative versus adjuvant systemic therapies only. Molecular targeted therapies were allowed; (III) studies reporting the outcomes of interest including overall, disease-free survival, R0 resection rate, perioperative complications and adverse effects of chemotherapy; (IV) we included randomized controlled trials (RCTs) and cohort studies. Abstracts of conference proceedings were allowed if sufficient information was reported on study design, participant demographics, interventions conducts and outcomes. Exclusion criteria were: (I) studies involving patients who had rectal cancer, or studies involving patients who had distant metastasis of colon cancer; (II) studies whose patients underwent chemoradiotherapy or did not undergo post-surgery chemotherapy; (III) studies which did not report the outcomes of interest; (IV) all articles that were not original studies, such as opinion pieces, reviews and commentaries.

\section{Data extraction and assessment of quality}

Three reviewers in blinded pairs independently reviewed included studies to perform data extraction using a predesigned sheet. We extracted the following data for each study: (I) general information: author, year of publication, title, source, country and language; (II) study characteristics: study design and duration of follow up; (III) the inclusion and exclusion criteria for study participants, size of each study arm, and baseline characteristics of each study arm; (IV) type of neoadjuvant and adjuvant chemotherapy used; (V) outcomes: hazard ratio (HR), odds ratio (OR), risk ratio (RR) and their 95\% confidence intervals (CIs). Quality assessment were independently assessed by two researchers using the Jadad Scale (13) for randomized trials and The Newcastle-Ottawa Scale (NOS) for cohort studies (14). All discrepancies were resolved with a senior author.

\section{Outcome measures}

The primary outcome was overall survival as reported using intention-to-treat analysis. Overall survival was defined as time from randomization to death. Secondary outcomes included: disease-free survival, which was reported as the 
time from randomization to disease recurrence or death; R0 resection rate, which was reported as rate of microscopically margin-negative resection; anastomotic leak which was defined as leakage of luminal contents after colorectal anastomosis; abdominal infection which was defined as surgical wound infection with or without intra-abdominal abscess. We expected the adverse effects of systemic therapies to be reported variably across trials and therefore not suitable for meta-analysis.

\section{Statistical analysis}

For the analysis of time to event data (overall and diseasefree survival), we estimated the HRs and 95\% CIs directly or indirectly from the given data (15). For each individual study, we extracted $\log$ HRs and their variances. If the figures were not given directly, methods of indirect determination were used (16). HRs can be estimated (under certain assumptions) from observed to expected event ratios (17). For the analysis of dichotomous data (R0 resection, anastomotic leak and abdominal infections), we estimated the OR or RR based on the number of patients in each treatment arm who experienced the event of interest and the number of patients assessed for the event of interest. Statistical heterogeneity of trial results was assessed by Cochran Q test and I-squared statistic. A Cochran Q test of $\mathrm{P}$ value higher than 0.10 and I-squared value of lower than $40 \%$ was interpreted as low level of heterogeneity $(18,19)$. There were too few studies included in the metaanalysis to conduct Egger's regression asymmetry test. Primary analyses were done with random effects model. All data synthesis was conducted using RevMan 5.3.

\section{Results}

\section{Search results}

In total, 2,461 English articles were identified according to the search strategy. Six hundred and eighty-seven were excluded after checking for duplicates with the literature management software Endnote X9. One thousand seven hundred and twenty-three studies were excluded after screening the titles and abstracts. Fifty-one studies were excluded after assessing the full text for eligibility. Eightyfour Chinese articles were identified according to the search strategy. Seventy-two studies were excluded after screening the titles and abstracts. Ten studies were excluded after assessing the full text for eligibility. In total, we found 7 eligible studies, with 2 originating from the United
Kingdom, 2 from China, 1 from the Netherlands, 1 from the United States of America, and 1 from France. A graphical summary of the article selection process is provided in Figure 1. In total, 2,006 patients underwent neoadjuvant treatment and 27,498 patients underwent adjuvant treatment for locally advanced colon cancer (Table 1).

\section{Oncological and surgical outcomes}

Overall survival significantly improved amongst 1,160 patients who received neoadjuvant chemotherapy compared to 27,042 patients who received adjuvant chemotherapy (20-23) (HR =0.76, 95\% CI: 0.65-0.89, $\mathrm{P}=0.0005$, Figure 2). Disease-free survival also significantly improved amongst 788 patients who received neoadjuvant chemotherapy compared to 444 patients who received adjuvant chemotherapy $(10,22,23)$ (HR $=0.74,95 \%$ CI: 0.58-0.95, $\mathrm{P}=0.02)$. The 995 patients who received neoadjuvant chemotherapy had a higher rate of R0 resection than 753 patients who received adjuvant chemotherapy, though results fell slightly short of statistical significance $(10,20,24,25)$ $(\mathrm{OR}=1.86,95 \% \mathrm{CI}: 0.95-3.62, \mathrm{P}=0.07$, Figure 3$)$. However, the authors of one of the included studies reported that their $\mathrm{R} 0$ resection rate could have been biased by the high percentage of neoadjuvant chemotherapy patients with unfavorable tumors (20). A sensitivity analysis showed that increase in R0 resection rate amongst the remaining included studies was statistically significant ( $\mathrm{OR}=2.48,95 \%$ CI: $1.15-5.39, \mathrm{P}=0.02)$.

\section{Surgical complications}

Rates of perioperative complications were similar between patients who underwent neoadjuvant chemotherapy and patients who underwent adjuvant chemotherapy. Comparative analysis of 238 patients in the neoadjuvant arm and 192 patients in the adjuvant arm revealed no significant differences in abdominal infection rates (22-24) (RR $=1.14,95 \%$ CI: 0.59-2.18, P=0.70, Figure 4). Similarly, there was no significant difference in rates of anastomotic leakage between 1,084 patients who underwent neoadjuvant chemotherapy and 844 patients who underwent adjuvant chemotherapy $(10,20,22-24,26)(\mathrm{RR}=0.83,95 \%$ CI: $0.53-$ $1.31, \mathrm{P}=0.42$, Figure 5).

\section{Adverse events from chemotherapy}

All studies found no significant difference in rates of 


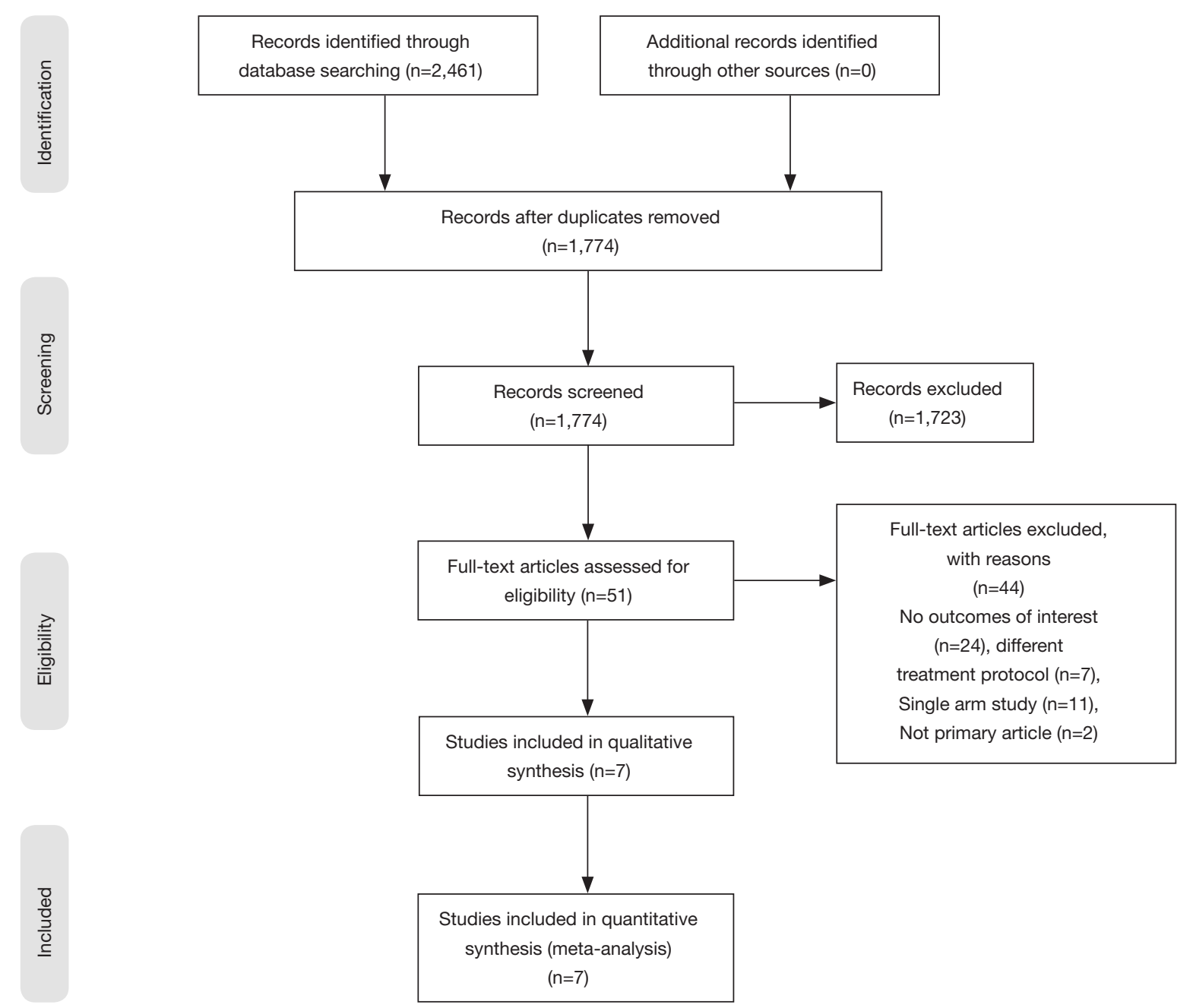

Figure 1 PRISMA flow diagram of included articles. PRISMA, Preferred Reporting Items for Systematic Reviews and Meta-Analyses.

chemotherapy adverse effects between patients who underwent neoadjuvant chemotherapy and patients who underwent adjuvant chemotherapy. Zhuang et al. (22) found no significant difference in rates of bowel irritation $(56 \%$ vs. $62 \%, \mathrm{P}>0.05)$, granulocytopenia ( $22 \%$ vs. $20 \%, \mathrm{P}>0.05)$, and thrombocytopenia $(16 \%$ vs. $14 \%, \mathrm{P}>0.05)$. Similarly, Song et al. (23) found no differences in rates of bowel irritation (55\% vs. 52.5\%, P>0.05), granulocytopenia (12.5\% vs. $10 \%, \mathrm{P}>0.05)$, and thrombocytopenia $(17.5 \%$ vs. $10 \%$, $\mathrm{P}>0.05$ ). FOXTROT (24) found no significant differences in rates of deep vein thrombosis $(2 \% v s .0 \%, \mathrm{P}=0.31)$, rash ( $3 \%$ vs. $0 \%, \mathrm{P}=0.21)$, neutropenia $(1 \%$ vs. $0 \%, \mathrm{P}=0.47)$, and bronchopneumonia ( $2 \%$ vs. $0 \%, \mathrm{P}=0.31)$. Similarly, Karoui et al. (25) found no significant differences in rates of thrombocytopenia ( $0 \%$ vs. $3 \%, \mathrm{P}>0.05)$, neutropenia $(17 \%$ vs. $24 \%, \mathrm{P}>0.05$ ), diarrhea ( $4 \%$ vs. $5 \%, \mathrm{P}>0.05$ ). Studies reported chemotherapy adverse effects with unsatisfactory congruency, hence reported findings could not be pooled for a meta-analysis.

\section{Discussion}

This is the first systematic review and meta-analysis of neoadjuvant chemotherapy versus adjuvant chemotherapy for locally advanced colon cancer, incorporating clinical trials with contemporary chemotherapy regimens from both English and Chinese literature. The review included a total of 2,006 patients undergoing neoadjuvant treatment and 27,498 patients undergoing adjuvant treatment. In all studies, patients who received neoadjuvant treatment also went on to receive postoperative adjuvant chemotherapy. Our analyses suggest potential benefit in using the 


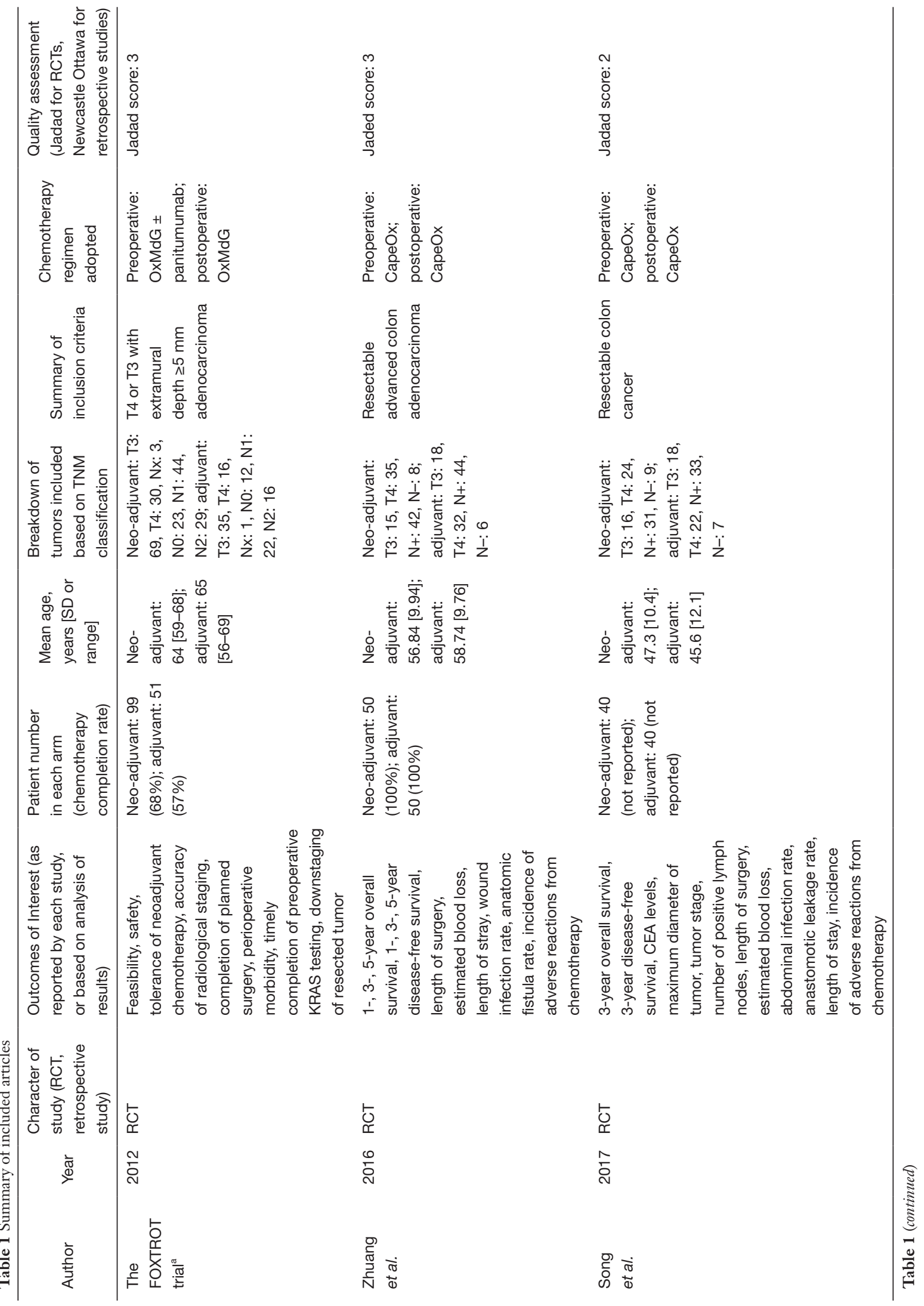




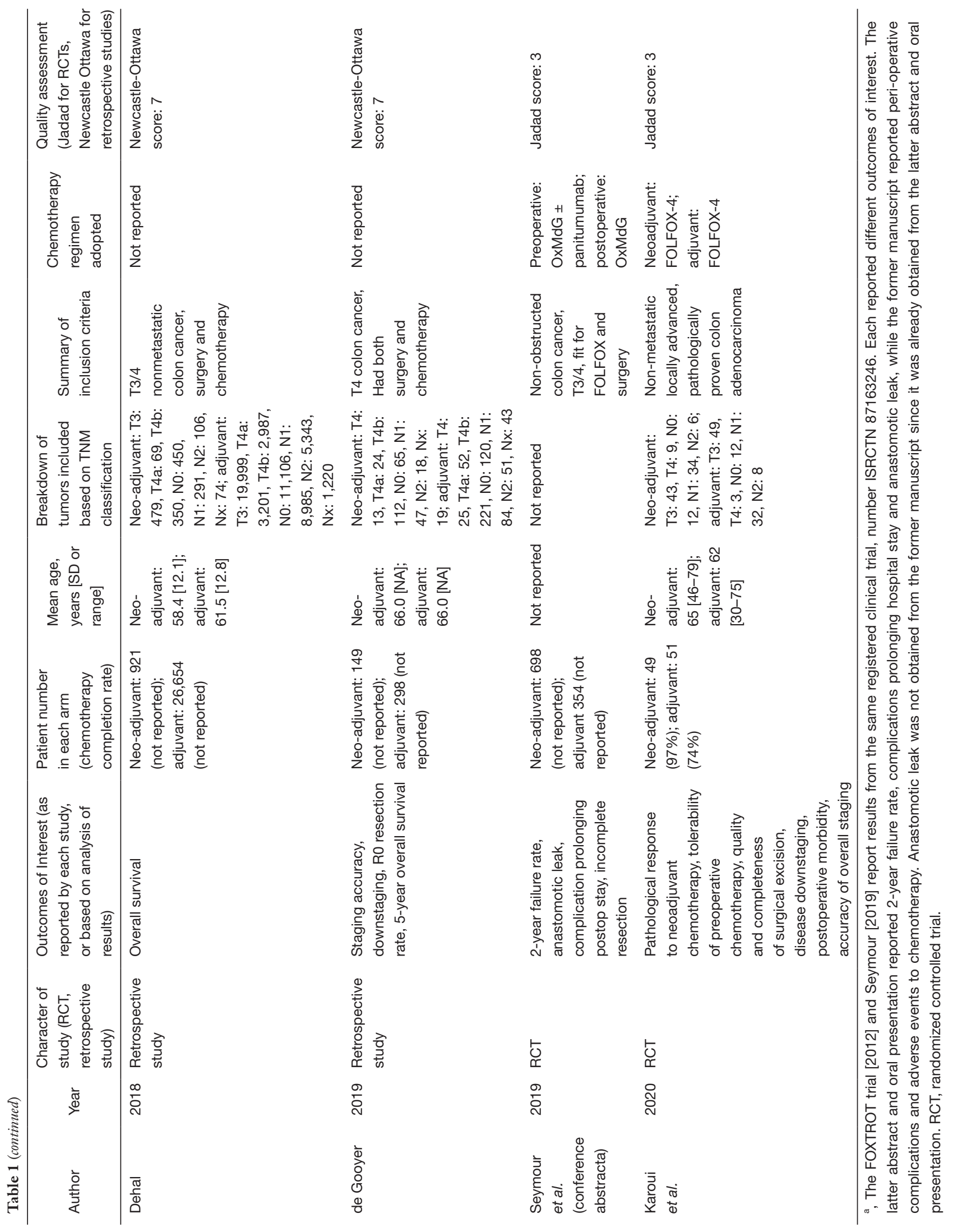




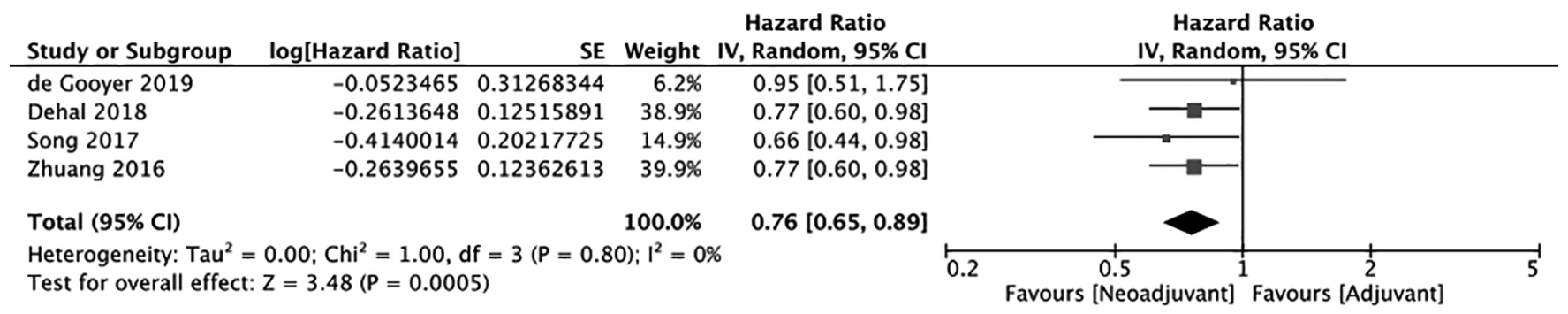

Figure 2 Meta-analysis of overall survival.

\begin{tabular}{|c|c|c|c|c|c|c|c|c|c|}
\hline Study or Subgroup & \multicolumn{2}{|c|}{ Experimental } & \multicolumn{2}{|c|}{ Control } & Weight & $\begin{array}{l}\text { Odds Ratio } \\
\mathrm{M}-\mathrm{H} \text {, Random, } 95 \% \mathrm{Cl}\end{array}$ & \multicolumn{3}{|c|}{$\begin{array}{c}\text { Odds Ratio } \\
\mathrm{M}-\mathrm{H}, \text { Random, } 95 \% \mathrm{Cl}\end{array}$} \\
\hline de Gooyer 2019 & 115 & 149 & 225 & 298 & $35.6 \%$ & $1.10[0.69,1.75]$ & & & \\
\hline FOXTROT Collaborative 2012 & 95 & 99 & 40 & 50 & $17.7 \%$ & $5.94[1.76,20.05]$ & & & \\
\hline Karoui 2019 & 46 & 49 & 48 & 51 & $11.8 \%$ & $0.96[0.18,4.99]$ & & & \\
\hline Seymour 2019 & 665 & 698 & 319 & 354 & $34.9 \%$ & $2.21[1.35,3.62]$ & & & \\
\hline Total $(95 \% \mathrm{Cl})$ & & 995 & & 753 & $100.0 \%$ & $1.86[0.95,3.62]$ & & & \\
\hline Total events & 921 & & 632 & & & & & & \\
\hline $\begin{array}{l}\text { Heterogeneity: } \operatorname{Tau}^{2}=0.27 ; C \\
\text { Test for overall effect: } Z=1.8\end{array}$ & $\begin{array}{l}i^{2}=8.98 \\
(P=0.0\end{array}$ & $d f=3$ & $(P=0.03$ & 3); $1^{2}=$ & $67 \%$ & & $\begin{array}{lc}0.05 & 0.2 \\
\text { Favours [Adjuvant] }\end{array}$ & $\begin{array}{c}5 \\
\text { Favours [Neoadjuvant] }\end{array}$ & 20 \\
\hline
\end{tabular}

Figure 3 Meta-analysis of R0 resection rate.

\begin{tabular}{|c|c|c|c|c|c|c|c|c|c|}
\hline Study or Subgroup & \multicolumn{2}{|c|}{ Experimental } & \multicolumn{2}{|c|}{ Control } & Weight & $\begin{array}{l}\text { Odds Ratio } \\
\mathrm{M}-\mathrm{H}, \text { Random, } 95 \% \mathrm{Cl}\end{array}$ & \multicolumn{3}{|c|}{$\begin{array}{l}\text { Odds Ratio } \\
\text { M-H, Random, } 95 \% \mathrm{Cl}\end{array}$} \\
\hline FOXTROT Collaborative 2012 & 13 & 99 & 4 & 51 & $30.6 \%$ & $1.78[0.55,5.76]$ & & $\square$ & \\
\hline Karoui 2019 & 2 & 49 & 3 & 51 & $12.6 \%$ & $0.68[0.11,4.26]$ & & & \\
\hline Song 2017 & 5 & 40 & 6 & 40 & $25.9 \%$ & $0.81[0.23,2.90]$ & - & & \\
\hline Zhuang 2016 & 7 & 50 & 6 & 50 & $30.9 \%$ & $1.19[0.37,3.84]$ & & & \\
\hline Total $(95 \% \mathrm{Cl})$ & & 238 & & 192 & $100.0 \%$ & $1.14[0.59,2.18]$ & & & \\
\hline Total events & 27 & & 19 & & & & & & \\
\hline \multicolumn{7}{|c|}{$\begin{array}{l}\text { Heterogeneity: } \text { Tau }^{2}=0.00 ; C^{2} i^{2}=1.13, d f=3(P=0.77) ; I^{2}=0 \% \\
\text { Test for overall effect: } Z=0.38(P=0.70)\end{array}$} & $\begin{array}{ccc}0.1 & 0.2 & 0.5 \\
& \text { Favours [Neoadjuvant] }\end{array}$ & Favours [Adjuvant] & 5 \\
\hline
\end{tabular}

Figure 4 Meta-analysis of abdominal infections.

\begin{tabular}{|c|c|c|c|c|c|c|c|c|}
\hline Study or Subgroup & \multicolumn{2}{|c|}{ Experimental } & \multicolumn{2}{|c|}{ Control } & Weight & $\begin{array}{c}\text { Risk Ratio } \\
\mathrm{M}-\mathrm{H}, \text { Random, } 95 \% \mathrm{Cl}\end{array}$ & \multicolumn{2}{|c|}{$\begin{array}{l}\text { Risk Ratio } \\
\text { M-H, Random, } 95 \% \mathrm{Cl}\end{array}$} \\
\hline de Gooyer 2019 & 8 & 149 & 9 & 298 & $24.0 \%$ & $1.78[0.70,4.51]$ & $\rightarrow$ & $\Longrightarrow$ \\
\hline FOXTROT Collaborative 2012 & 5 & 98 & 2 & 51 & $8.1 \%$ & $1.30[0.26,6.47]$ & & \\
\hline Karoui 2019 & 1 & 49 & 1 & 51 & $2.8 \%$ & $1.04[0.07,16.18]$ & & \\
\hline Seymour 2019 & 22 & 698 & 20 & 354 & $59.5 \%$ & $0.56[0.31,1.01]$ & & \\
\hline Song 2017 & 1 & 40 & 1 & 40 & $2.8 \%$ & $1.00[0.06,15.44]$ & & \\
\hline Zhuang 2016 & 1 & 50 & 1 & 50 & $2.8 \%$ & $1.00[0.06,15.55]$ & & \\
\hline Total $(95 \% \mathrm{Cl})$ & & 1084 & & 844 & $100.0 \%$ & $0.83[0.53,1.31]$ & & \\
\hline Total events & 38 & & 34 & & & & & \\
\hline \multicolumn{7}{|c|}{$\begin{array}{l}\text { Heterogeneity: } \text { Tau }^{2}=0.00 ; C^{2} i^{2}=4.66, d f=5(P=0.46) ; I^{2}=0 \% \\
\text { Test for overall effect: } Z=0.80(P=0.42)\end{array}$} & 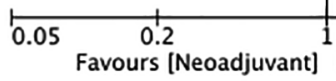 & $\begin{array}{c}5 \\
\text { Favours [Adjuvant] }\end{array}$ \\
\hline
\end{tabular}

Figure 5 Meta-analysis of anastomotic leak. 
neoadjuvant approach compared to adjuvant chemotherapy. More importantly, there were no differences in surgical complications. While the efficacy of neoadjuvant chemotherapy is still under investigation for colon cancer, its use has been well established for the treatment of rectal cancer-current guidelines recommend neoadjuvant chemotherapy for all T3 and T4 tumors $(27,28)$. This is in part due to the significant decrease in local recurrence rates associated with use of neoadjuvant chemotherapy $(29,30)$. Such findings are also consistent with the rapidly emerging use of total neoadjuvant therapy-a recent systematic review and meta-analysis concluded that patients with rectal cancer receiving total neoadjuvant therapy had a better disease-free survival and overall survival (31).

In line with these studies, our study found that overall survival \& disease-free survival improved in patients receiving neoadjuvant chemotherapy for colon cancer. The improvement in overall survival and disease-free survival for patients treated with neoadjuvant therapy could be due to improved R0 resection rates, which is in line with existing findings regarding the importance of $\mathrm{R} 0$ resection rate in colorectal cancer (32,33). As discussed earlier, while one study did not find a significant improvement in $\mathrm{R} 0$ resection rate, the authors suspected this was due to significant percentage of patients with unfavorable tumors in the neoadjuvant arm (20). Our findings regarding R0 resection rate further support the notion of the clinical usefulness of neoadjuvant chemotherapy for locally advanced colon cancer.

Neoadjuvant chemotherapy has several additional potential advantages over adjuvant chemotherapy. Firstly, it reduces reliance on adjuvant chemotherapy to achieve treatment efficacy. This is key, as delays in post-operative chemotherapy arising from surgical complications has been shown to result in poorer overall survival (34-36). A recent randomized trial comparing different types of neoadjuvant chemotherapy in colon cancer found low recurrence rate and high disease-free survival in a subgroup of patients for whom adjuvant chemotherapy was omitted altogether (37). Secondly, in studies based largely on metastatic colon cancer $(38,39)$, pre-operative chemotherapy allows for patient selection in terms of tumor biology and possibly prevents unnecessary operations for patients with poor response (21). Lastly. the neoadjuvant approach also paves the route for the design of clinical trials incorporating novel agents to allow for quicker in vivo and early read out of efficacy. Compared to breast cancer and lung cancer, which have had a rapid increase in survival due to the identification of novel targets and pathways, colon cancer treatment has significantly lagged behind in the field of precision oncology $(40,41)$.

Incorporation of neoadjuvant approaches in combination with biomarker selected strategies will advance the field of precision oncology in colon cancer significantly. In fact, the clinical usefulness of neoadjuvant chemotherapy may be increased in pT4 subgroup patients. One study reported a significant improvement in overall survival only within the pT4b subgroup (21). Two other studies that reported significant improvement in disease-free survival had relatively high proportion of pT4 patients $(22,23)$. However in contrast, the FOXTROT trial that had high proportion of pT3 patients reported no significant difference in diseasefree survival (10). While we acknowledge that the data is premature, it does suggest that neoadjuvant chemotherapy would likely benefit the pT4 patients most and might be the distinction for tumor board groups to decide on whom to select for future neoadjuvant therapy versus adjuvant therapy. Thus far, the application of neoadjuvant treatment has however been limited by concerns of inaccurate radiological staging resulting in overestimation of actual pathological stage and inappropriate chemotherapy for lowrisk patients (42), and concerns of complications leading to poorer outcomes such as tumor perforation, bleeding and obstruction.

Our study has established that these concerns may be unfounded, particularly with respect to surgical outcomes. We assessed perioperative morbidity, which is another important aspect of neoadjuvant chemotherapy. In terms of post-operative complications, it is estimated that about $20 \%$ of patients experience serious complications related to adjuvant chemotherapy (43). Although a meta-analysis could not be comprehensively performed for all postoperative complications in our study, it does seem that patients who underwent neoadjuvant chemotherapy did not experience serious complications in excess of $20 \%$ from the studies analyzed. Furthermore, from our results, the similar rates of anastomotic leak and abdominal infection supports the promising use of neoadjuvant treatment. Future studies for neoadjuvant chemotherapy for locally advanced colon cancer will mature in the next decade. Other than the FOXTROT trial, the PRODIGE 22-ECKINOXE trial (26) is a multicenter randomized phase II trial where patients with T3-T4 and/or N2 were randomized to either preoperative FOLFOX followed by surgery followed by postoperative FOLFOX, or surgery followed by FOLFOX which is expected to conclude by 2021 . Two other ongoing 
trials registered in clinicialtrials.gov include one from Denmark (44) and the other from South Korea (45).

\section{Limitations}

There are some limitations to our current study. The review is limited inherently by the amount of studies included. That said, a total of 29,504 patients were included in our analyses, circumnavigating this problem and providing preliminary evidence substantiating the use of neoadjuvant in locally advanced colon cancer. Additionally, the practicality of blinding participants in the trials would also affect the rigor in quality assessment.

\section{Conclusions}

In conclusion, this meta-analysis is timely in an emerging era of total neoadjuvant treatment for colorectal cancer. The improved survival and no difference in post-operative complications with neoadjuvant chemotherapy treatment will impact tumor board discussions for non-metastatic locally advanced colon cancer. With the advances made in standard and targeted therapy, there is a foreseeable trend of increased adoption of neoadjuvant chemotherapy for curable colon cancers in the next decade.

\section{Acknowledgments}

The authors thank Ms. Annelissa Chin, senior medical librarian at Yong Loo Lin School of Medicine, National University Singapore, for her assistance in deriving the search strategy.

Funding: None.

\section{Footnote}

Reporting Checklist: The authors present the article in accordance with the PRISMA reporting checklist. Available at http://dx.doi.org/10.21037/jgo-20-220

Conflicts of Interest: All authors have completed the ICMJE uniform disclosure form (available at http://dx.doi. org/10.21037/jgo-20-220). The authors have no conflicts of interest to declare.

Ethical Statement: The authors are accountable for all aspects of the work in ensuring that questions related to the accuracy or integrity of any part of the work are appropriately investigated and resolved.

Open Access Statement: This is an Open Access article distributed in accordance with the Creative Commons Attribution-NonCommercial-NoDerivs 4.0 International License (CC BY-NC-ND 4.0), which permits the noncommercial replication and distribution of the article with the strict proviso that no changes or edits are made and the original work is properly cited (including links to both the formal publication through the relevant DOI and the license). See: https://creativecommons.org/licenses/by-nc-nd/4.0/.

\section{References}

1. Bray F, Ferlay J, Soerjomataram I, et al. Global cancer statistics 2018: GLOBOCAN estimates of incidence and mortality worldwide for 36 cancers in 185 countries. CA Cancer J Clin 2018;68:394-424.

2. Benson AB, Venook AP, Al-Hawary MM, et al. NCCN guidelines insights: colon cancer, version 2.2018. J Natl Compr Canc Netw 2018;16:359-69.

3. Al-Batran SE, Homann N, Schmalenberg H, et al. Perioperative chemotherapy with docetaxel, oxaliplatin, and fluorouracil/leucovorin (FLOT) versus epirubicin, cisplatin, and fluorouracil or capecitabine (ECF/ECX) for resectable gastric or gastroesophageal junction (GEJ) adenocarcinoma (FLOT4-AIO): a multicenter, randomized phase 3 trial. J Clin Oncol 2017;35:4004.

4. van Hagen P, Hulshof MC, van Lanschot JJ, et al. Preoperative chemoradiotherapy for esophageal or junctional cancer. N Engl J Med 2012;366:2074-84.

5. Sauer R, Liersch T, Merkel S, et al. Preoperative versus postoperative chemoradiotherapy for locally advanced rectal cancer: results of the German CAO/ARO/AIO-94 randomized phase III trial after a median follow-up of 11 years. J Clin Oncol 2012;30:1926-33.

6. Rastogi P, Anderson SJ, Bear HD, et al. Preoperative chemotherapy: updates of National Surgical Adjuvant Breast and Bowel Project Protocols B-18 and B-27. J Clin Oncol 2008;26:778-85.

7. Jakobsen AKM, Andersen F, Fischer A, et al. A markerdriven phase II trial of neoadjuvant chemotherapy in locally advanced colon cancer. J Clin Oncol 2014;32:3621.

8. Arredondo J, Baixauli J, Pastor C, et al. Mid-term oncologic outcome of a novel approach for locally advanced colon cancer with neoadjuvant chemotherapy and surgery. Clin Transl Oncol 2017;19:379-85.

9. Aisu N, Yoshida Y, Komono A, et al. Perioperative 
chemotherapy with S-1 plus oxaliplatin (SOX) for stage III colorectal cancer patients. J Clin Oncol 2016;34:603.

10. Seymour MT, Morton D. FOxTROT: an international randomised controlled trial in 1052 patients (pts) evaluating neoadjuvant chemotherapy (NAC) for colon cancer. J Clin Oncol 2019;37:3504.

11. Moher D, Liberati A, Tetzlaff J, et al. Preferred reporting items for systematic reviews and meta-analyses: the PRISMA statement. PLoS Med 2009;6:e1000097.

12. Cohen JF, Korevaar DA, Wang J, et al. Should we search Chinese biomedical databases when performing systematic reviews? Syst Rev 2015;4:23.

13. Jadad AR, Moore RA, Carroll D, et al. Assessing the quality of reports of randomized clinical trials: is blinding necessary? Control Clin Trials 1996;17:1-12.

14. Wells GA, Shea B, O'Connell D, et al. The NewcastleOttawa Scale (NOS) for assessing the quality of nonrandomized studies in meta-analyses. Ottawa Hospital Research Institute, 2019. Available online: http://www. ohri.ca/programs/clinical_epidemiology/oxford.asp

15. Altman DG. Systematic reviews of evaluations of prognostic variables. BMJ 2001;323:224-8.

16. Altman DG, Bland JM. How to obtain the confidence interval from a P value. BMJ 2011;343:d2090.

17. Tierney JF, Stewart LA, Ghersi D, et al. Practical methods for incorporating summary time-to-event data into metaanalysis. Trials 2007;8:16.

18. Fletcher J. What is heterogeneity and is it important? BMJ 2007;334:94-6.

19. DerSimonian R, Laird N. Meta-analysis in clinical trials. Control Clin Trials 1986;7:177-88.

20. de Gooyer JM, Verstegen MG, 't Lam-Boer J, et al. Neoadjuvant chemotherapy for locally advanced T4 colon cancer: a nationwide propensity-score matched cohort analysis. Dig Surg 2020;37:292-301.

21. Dehal A, Graff-Baker AN, Vuong B, et al. Neoadjuvant chemotherapy improves survival in patients with clinical T4b colon cancer. J Gastrointest Surg 2018;22:242-9.

22. Zhuang $Y$, Lin J, Huang M, et al. Neoadjuvant chemotherapy for resectable locally advanced colon carcinoma: a prospective case controlled trial. Lingnan Modern Clinics in Surgery 2016;16:266-9.

23. Song T, Wang D, Xiao G, et al. Clinical efficacy of capecitabine combined with oxaliplation on preoperative neoadjuvant chemotherapy for colorectal cancer. Journal of Guizhou Medical University 2017;42:1107-10.

24. Foxtrot Collaborative Group. Feasibility of preoperative chemotherapy for locally advanced, operable colon cancer: the pilot phase of a randomised controlled trial. Lancet Oncol 2012;13:1152-60.

25. Karoui M, Rullier A, Piessen G, et al. Perioperative FOLFOX 4 versus FOLFOX 4 plus cetuximab versus immediate surgery for high-risk stage II and III colon cancers: a phase II multicenter randomized controlled trial (PRODIGE 22). Ann Surg 2020;271:637-45.

26. Karoui M, Rullier A, Luciani A, et al. Neoadjuvant FOLFOX 4 versus FOLFOX 4 with Cetuximab versus immediate surgery for high-risk stage II and III colon cancers: a multicentre randomised controlled phase II trial--the PRODIGE 22--ECKINOXE trial. BMC Cancer 2015;15:511.

27. Benson AB, Venook AP, Al-Hawary MM, et al. Rectal cancer, version 2.2018, NCCN clinical practice guidelines in oncology. J Natl Compr Canc Netw 2018;16:874-901.

28. Glynne-Jones R, Wyrwicz L, Tiret E, et al. Rectal cancer: ESMO Clinical Practice Guidelines for diagnosis, treatment and follow-up. Ann Oncol 2018;29:iv263.

29. Bosset JF, Collette L, Calais G, et al. Chemotherapy with preoperative radiotherapy in rectal cancer. N Engl J Med 2006;355:1114-23.

30. Gérard JP, Conroy T, Bonnetain F, et al. Preoperative radiotherapy with or without concurrent fluorouracil and leucovorin in T3-4 rectal cancers: results of FFCD 9203.J Clin Oncol 2006;24:4620-5.

31. Petrelli F, Trevisan F, Cabiddu M, et al. Total neoadjuvant therapy in rectal cancer: a systematic review and metaanalysis of treatment outcomes. Ann Surg 2020;271:440-8.

32. Bhangu A, Ali SM, Darzi A, et al. Meta-analysis of survival based on resection margin status following surgery for recurrent rectal cancer. Colorectal Dis 2012;14:1457-66.

33. Khan MA, Hakeem AR, Scott N, et al. Significance of R1 resection margin in colon cancer resections in the modern era. Colorectal Dis 2015;17:943-53.

34. Bayraktar UD, Chen E, Bayraktar S, et al. Does delay of adjuvant chemotherapy impact survival in patients with resected stage II and III colon adenocarcinoma? Cancer 2011;117:2364-70.

35. Glimelius B, Dahl O, Cedermark B, et al. Adjuvant chemotherapy in colorectal cancer: a joint analysis of randomised trials by the Nordic Gastrointestinal Tumour Adjuvant Therapy Group. Acta Oncol 2005;44:904-12.

36. Des Guetz G, Nicolas P, Perret GY, et al. Does delaying adjuvant chemotherapy after curative surgery for colorectal cancer impair survival? A meta-analysis. Eur J Cancer 2010;46:1049-55.

37. Jakobsen A, Andersen F, Fischer A, et al. Neoadjuvant 
chemotherapy in locally advanced colon cancer. A phase II trial. Acta Oncol 2015;54:1747-53.

38. Mentha G, Majno PE, Andres A, et al. Neoadjuvant chemotherapy and resection of advanced synchronous liver metastases before treatment of the colorectal primary. Br J Surg 2006;93:872-8.

39. Adam R, Pascal G, Castaing D, et al. Tumor progression while on chemotherapy: a contraindication to liver resection for multiple colorectal metastases? Ann Surg 2004;240:1052-61; discussion 1061-4.

40. Sundar R, Chénard-Poirier M, Collins DC, et al. Imprecision in the era of precision medicine in non-small cell lung cancer. Front Med (Lausanne) 2017;4:39.

41. Sundar R, Tan IBH, Chee CE. Negative predictive biomarkers in colorectal cancer: PRESSING ahead. J Clin
Oncol 2019;37:3066-8.

42. Zhou Z, Nimeiri HS, Benson AB 3rd. Preoperative chemotherapy for locally advanced resectable colon cancer - a new treatment paradigm in colon cancer? Ann Transl Med 2013;1:11.

43. Merkow RP, Bentrem DJ, Mulcahy MF, et al. Effect of postoperative complications on adjuvant chemotherapy use for stage III colon cancer. Ann Surg 2013;258:847-53.

44. Neoadjuvant chemotherapy versus standard treatment in patients with locally advanced colon cancer. Available online: https://ClinicalTrials.gov/show/NCT01918527

45. Neoadjuvant FOLFOX chemotherapy for patients with locally advanced colon cancer. Available online: https:// ClinicalTrials.gov/show/NCT03426904
Cite this article as: Cheong CK, Nistala KRY, $\mathrm{Ng}$ CH, Syn N, Chang HSY, Sundar R, Yang SY, Chong CS. Neoadjuvant therapy in locally advanced colon cancer: a meta-analysis and systematic review. J Gastrointest Oncol 2020;11(5):847-857. doi: 10.21037/jgo-20-220 


\section{Supplementary}

\section{MEDLINE search strategy}

1. Colorectal Neoplasms/

2. (Colo* adj3 (carcinom* or neoplas* or adenocarcinom* or cancer* or tumour* or adenoma* or malignan*)).tw.

3. 1 or 2

4. exp Chemotherapy, Adjuvant/

5. Neo-adjuvant.tw.

6. Neo adjuvant.tw.

7. FOLFOX.tw.

8. XELOX.tw.

9. CAPOX.tw.

10. 5-fu.tw.

11. Oxaliplatin.tw.

12. Fluorouracil.tw.

13. 5-fluorouracil.tw.

14. Capecitabine.tw.

15. Leucovorin.tw.

16. 4 or 5 or 6 or 7 or 7 or 9 or 10 or 11 or 12 or 13 or 14 or 15

17. exp laparoscopy/ or laparoscop* .mp. or (laparoscop* adj3 (surg* or operat* or procedure or resecti* or Colectomy)).tw.

18. ((robot* ${ }^{*}$ or Open or Convention*) adj5 (surg* or operat* or procedure or resecti* or Colectomy)).tw

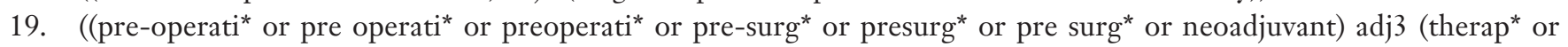
treatment* ${ }^{*}$ chemotherap* or down staging or down stage)).tw.

20. 16 and 19

21. (surg* or operat $^{*}$ or procedure or resecti* or colectomy).tw.

22. 3 and 21

23. 17 or 18 or 22

24. 3 and 20 and 23 